\title{
Research Of Piezo-resistive And Piezoelectric Sensor
}

\author{
Sanming Feng ${ }^{\mathrm{a}}$, Wenjie Tian $^{\mathrm{b},{ }^{,}}$, Bo $\mathrm{Ma}^{\mathrm{c}}$ \\ Beijing Information Science and Technology University \\ 100101,Beijing,China \\ a996613627@qq.com, btwjkm2008@163.com, '820265449@qq.com \\ * Corresponding Author
}

Keywords: pressure sensor; tantalum nitride; PVDF; circuit

Abstract. The basic principle and application of piezo-resistive and piezoelectric are expounded. Also the method of deposition of tantalum nitride on the glass to fabricate piezo-resistive pressure sensor, and the method of a plurality of piezoelectric thin film in parallel to form a high sensitivity sensor are introduced. Besides, a power supply circuit and corresponding processing circuit are designed. The output voltage of the piezo-resistive pressure sensor is liner with respect to the pressure. The output of the charge is liner with the pressure of piezoelectric sensor first, then tends to saturation and presents less change. The sensitivity of piezoelectric sensor is up to $10^{3}$ orders of magnitude, with a higher sensitivity.

\section{Introduction}

Sensor is the first threshold of the information system, which is responsible for collecting information. Sensor technology, communication technology and computer technology have become the three pillars of modern information technology ${ }^{[1]}$, is an important basis for the information industry, so the importance of sensor technology is self-evident.

At present, the sensor industry in China is small, and the application scope is narrow. Therefore, we need to change the concept, the research and development of the sensor is developed by a single sensor, which is transformed into a new type of high integration. 30 years of reform and opening up, China's sensor technology and its industry has made great progress, the main performance in: the establishment of the National Key Laboratory of sensor technology, micro national key laboratories, national sensing technology engineering center and other research and development base; MOEMS, MEMS and other research projects included in the national high-tech development focus ${ }^{[2,3]}$.

In 1945, Smith discovered the pressure resistance effect of silicon and germanium, that is, when there is an external force acting on the semiconductor material, the resistance will change significantly. According to this principle, the pressure sensor is a strain resistance film on the metal film, the force signal into electrical signal measurement ${ }^{[4]}$. The minimum size of this stage is about $1 \mathrm{~cm}$. With the development of material technology, micro machining technology and microelectronic technology, much research work has been carried out, and the research results have been put into commercial field. Which has attracted worldwide attention, has made great progress.

\section{Pressure Resistance Type Pressure Sensor}

\section{Principles}

When the force acting on the material, the crystal lattice deformation, so that the carrier from one to the other to the valley scattering, causing the carrier to change the migration rate, the carrier's longitudinal and lateral, so that the resistivity of the material change ${ }^{[5]}$.The resistive element 
converts mechanical quantity sensor is strain input into resistance changes. The input resistance of the converter's strain $\varepsilon=\Delta L / L$, the length of the relative variation, it is a dimensionless relative value. Usually epsilon $=10^{-6}$ for a micro strain. The output resistance of the converter's relative is variation of $\Delta R / R$ resistance value. The change of the resistance of the metal resistance wire is mainly based on the structure size.

According to semiconductor piezoresistive effect of $\Delta \rho / \rho=\pi \sigma$ and $\sigma=E \varepsilon$ : $\sigma$ is a stress $(F / s)$; $\pi$ is the pressure drag coefficient and $\pi=(40 \sim 80) \times 10^{-11} \mathrm{~m}^{2} / \mathrm{N}, E$ is the elastic modulus, $E=1.67 \times$ $10^{11} \mathrm{~N} / \mathrm{m}^{2}$, so the relative change of resistivity $(\Delta \rho / \rho)=\pi E \varepsilon$.

$$
\frac{\mathrm{d} R}{R}=\frac{d l}{l}-\frac{d A}{A}+\frac{d p}{p}, \frac{\mathrm{d} R}{R}=\varepsilon-2 \mu \varepsilon+\pi E \varepsilon
$$

$\mu$ is Poisson's ratio of the material, $\varepsilon=\Delta L / L$, the relative variation of the length of material. Semiconductor material is proportional to the change in the relative change of the resistance of the semiconductor material and the length of the semiconductor material.

\section{Material Selection}

Tantalum nitride is a kind of excellent piezo-resistive materials, its structure is complex, known phase of up to 11 , such as hexagonal $\mathrm{Ta}_{2} \mathrm{~N}$, face centered cubic structure $\mathrm{Ta}_{4} \mathrm{~N}_{5}$, hexagonal structure , tetragonal structure $\mathrm{Ta}_{4} \mathrm{~N}_{5}$, orthorhombic structure of $\mathrm{Ta}_{3} \mathrm{~N}_{5}$ and so on. Melting point of 3000 degrees Celsius, low temperature coefficient of resistance, excellent hardness, wear resistance ${ }^{[3]}$. The maximum hardness of $220 \sim 180 \mu \Omega \cdot \mathrm{cm}$, TCR $<-50 \times 10^{-6} /{ }^{\circ} \mathrm{C}$, bulk tin nitride material can only reach $1000 \mathrm{kgf} / \mathrm{mm}^{2}$. The preparation method of tantalum nitride materials mainly including magnetron sputtering deposition, ion beam enhanced deposition, chemical vapor deposition, pulsed laser deposition, liquid phase reaction method, and so on.

The new thin film system structure is made by reactive plasma sputtering on a small 7059 Corelle glass substrate deposited on tantalum nitride thin films. It can measure the change of pressure in the range of $10^{-3}$ to $10^{5} \mathrm{~Pa} .14 \times 17 \times 0.3 \mathrm{~mm} 7059$ Corelle glass is first placed in acetone, with ultrasonic cleaning, and then put into alcohol wash. In argon sputtering method complete deposition, the deposition of thin films of glass pieces of side length of $2 \mathrm{~mm}$ slices and use it for diaphragm vacuum gauge small sensitive element, the parallel gap pressure welding method, the second root diameter $0.08 \mathrm{~mm}$ copper wire welded to the gold electrode, the sensitive element.

\section{Constant Current Source Power Supply Circuit Design}

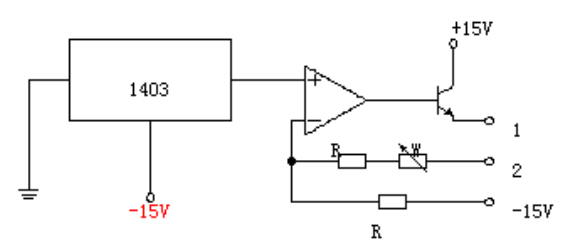

Fig. 1 power supply circuit diagram of constant current source

The constant current source power supply circuit uses dual power supply to avoid commo n mode interference, the 2 port current $I=2.5 / R$. Its stability depends on the stability of the reference voltage source and the resistance $R$. 


\section{Processing Circuit Design}

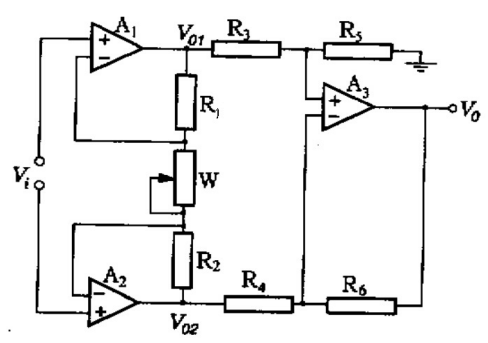

Fig. 2 processing circuit diagram

The full range output signal of the piezo-resistive sensor is unequal to $70-350 \mathrm{mv}$, and $\mathrm{t}$ he output impedance is very high, which requires that the amplifier circuit must have a hig her input impedance, and do not absorb the current from the output of the sensor so as no $t$ to damage the working state of the sensor. Figure $A_{2}, A_{1}$, the first stage in phase parallel differential amplifier, the amplifier output

$$
V_{0}{ }^{\prime}=V_{01}-V_{02}=\left[1+\left(\frac{R_{1}+R_{2}}{W}\right)\right] V_{\mathrm{i}} \text {. }
$$

$\mathrm{A}_{1}, \mathrm{~A}_{2}$ input is not absorbed current, and the circuit structure is symmetrical, drift and offs et are offset each other, has the ability to suppress common mode signal interference. $\mathrm{A}_{3} \mathrm{c}$ onstitutes the second stage differential amplifier, and the increase of the amplifier, to effecti vely suppress the common mode signal interference, to make the circuit $R_{5}=R_{6}=R_{f}, R_{3}=R_{4}=R$, the total output of the amplifier is

$$
V_{0}=-\left(\frac{R_{f}}{R}\right) V_{0}{ }^{\prime}=-\left(\frac{R_{f}}{R}\right)\left[1+\left(\frac{R_{1}+R_{2}}{W}\right)\right] V_{i} \text {. }
$$

Adjust the potentiometer $\mathrm{W}$, can change the amplifier gain, $R_{1}=R_{2}=R$, then

$$
V_{0}=-\frac{R_{f}}{R}\left(1+\frac{2 R}{W}\right) V_{i}
$$

In the formula, $V_{i}$ is determined by the constant current source and the resistance of the sensing element. The pressure is sensitive to the sensitive material. The current is constant and the $V_{i}$ changes, then the $V_{0}$ changes.

\section{Test Results}

Test, set 7 different sets of pressures acting on the sensitive element value, measured seven groups corresponding output voltage value, the result shown in Fig.3, seen from the figure the pressure value is approximately the size of the output voltage linearly relationship.

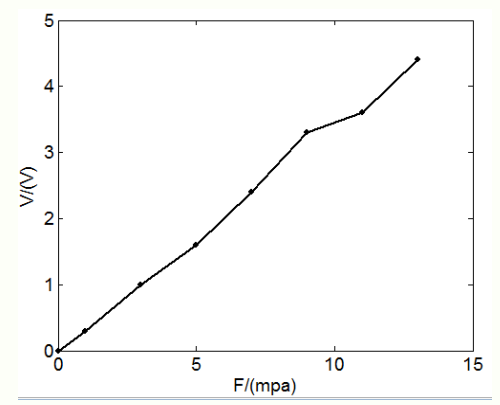

Fig. 3 the output voltage and pressure diagram 


\section{The Piezoelectric Pressure Sensor}

\section{The Basic Principles}

Principle: When some dielectric is deformed by external forces in a certain direction, its interior will produce polarization phenomenon, while the positive and negative charges will appear on its two opposite surfaces. When the force is removed, it will return to the uncharged state. This phenomenon is called piezoelectric effect. The polarity of the charge also changes along with the direction of the force. The sensors developed by the dielectric piezoelectric effect are called piezoelectric sensors. Piezoelectric type pressure sensor has been widely used in biomedical measurements, for example, ventricular catheter microphone is by the pressure electric sensor made of pressure electric pressure sensor is commonly used in dynamic pressure, so piezoelectric pressure sensor application is very large.

\section{The Selection Of Materials}

Jinzhou Branch letter Electronic Materials Ltd. supported technology by the Tsinghua University successfully achieves in the localization of production in PVDF piezoelectric film. It has the unique dielectric effect, piezoelectric effect and thermoelectric effect. Compared with a conventional piezoelectric, it has some advantages such as wide frequency response, wide dynamic range, high sensitivity in conversion of power and electric, high mechanical strength properties and the acoustic impedance easily matched. It also has advantages such as light weight, soft and not brittle, impact resistance and is not easily contaminated by water and chemicals. It is easy to be made to any shape and size of the different sheet or tube. It is widely used in the field of mechanical technology and a new material to improve pressure transducer dynamic sensor.

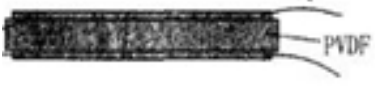

Fig. 4 Schematic diagram of strain structure of PVDF thin film

\section{The Connection Of PVDF Module}

Parallel connection: two PVDF module bond as the opposite direction of polarization. The negative charges concentrate in the middle negative electrode plate, while the positive charges are in the positive electrode plates at both ends. At this time it is the equivalent of two capacitors connected in parallel and the charge and capacity in output electrode plate will be doubled. If there are $n$ modules are connected in parallel, the total output of the charge at this time will increase $n$ times. The sensitivity of charges also increases $\mathrm{n}$ times. The voltage sensitivity is the same as a single module. Production process can refer to the patent that has been applied. The charges arising from the $\mathrm{n}$ modules in parallel are: $Q_{\mathrm{X}}=\mathrm{nd}_{11} F_{\mathrm{X}} \quad\left(\mathrm{d}_{11}\right.$ as a piezoelectric modulus of PVDF module, $F_{\mathrm{X}}$ as the force of the vertical direction). When the piezoelectric elements made of a piezoelectric material subject to pressure, the amount of charge and the force have a linear relationship: $Q=K S P$. $Q$ is a charge amount, $K$ is the piezoelectric constant, $S$ is the role of the area, $P$ is the pressure ${ }^{[5]}$. The value of the measured pressure can be known by measuring the amount of charge.

\section{The Design Of Processing Circuit}

PVDF film generates a weak charge signal and the film resistance is large. Its charge signal is small and easy escapes. So a suitable front circuit is needed to design to convert the weak charge signal into a voltage signal with a higher Signal to Noise Ratio and certain immunity. The pre-circuit composed by the pre-linear charge amplifier (output $U_{2}$ ), the secondary voltage amplifier 
(output $U_{3}$ ) and end-stage low-pass filter (output $U_{4}$ ), as shown in Fig. 5.

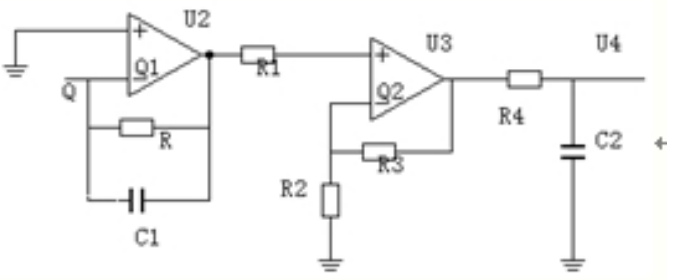

Fig.5 PVDF pressure sensor processing circuit diagram

The effects of linear charge amplifier are: (1) It can match with PVDF sensor impedance and transform the high-impedance input to low output impedance; (2) It can convert the weak charge signal into a voltage signal and amplify it. The main element of a charge amplifier is the capacitive that is inversely proportional to the voltage: $U_{2}=-Q / C$. Voltage amplifier amplifies the voltage signal output from the charge amplifier and provides different gains. The low-pass filter circuit is composed by $R_{4}$ and $C_{2}$ and selects cut-off frequency by adjusting $R_{4}$ and $C_{2}$.

$$
U_{3}=\left(1+\frac{R_{3}}{R_{2}}\right) U_{2}, U_{4}=\frac{U_{3}}{1+R_{4} \mathrm{j} \omega C_{2}}
$$

Because when the piezoelectric elements made of a piezoelectric material subject to pressure, the amount of charge and the force have a linear relationship: $Q=K S P$. In this study, the measured pressure can be known by the charge amount that is measured by the output voltage.

\section{The Results Of PVDF Piezoelectric Sensor Test}

At the temperature of $20{ }^{\circ} \mathrm{C}$, the experiments carried out 14 tests under the impact of the one-dimensional strain. It obtains corresponding values and sensitivity of 14 groups under the force. The results after scaling are shown in Fig.6. The pattern obtained in tests is approximately linear relationship and then becomes constant. The cause of this result may that the charge accumulation of the piezoelectric film at high pressure saturates and the charge amount becomes large with the decrease of force change rate.

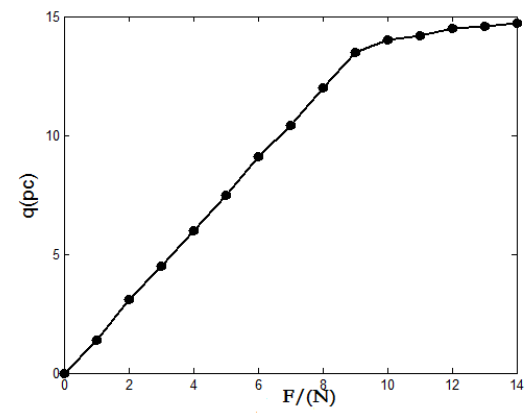

Fig. 6 PVDF test results

\section{The Conclusion}

By comparing the two most commonly production of pressure sensors, many similarities and differences can be found: (1) The input and the output of piezo-resistive sensors are approximately linear. When the pressure is small, the output of piezoelectric sensors is approximately linear relationship. When the pressure is too large, the output will tend constant; (2) They are measured by converting the force to the changes in electricity; (3) The piezo-resistive is directly tested in accordance with the change in resistance of the sensitive material, while the piezoelectric was tested according to the charge amount produced by the piezoelectric effect. 


\section{Acknowledgement}

This work was supported by National Natural Science Foundation of China (60968001), Beijing Natural Science Foundation (4122030), Beijing natural science foundation of key projects (B) (KZ201511232037), Key Laboratory of Modern Measurement \& Control Technology (Beijing Information Science \& Technology University), Ministry of Education

\section{Reference}

[1] Guangyu Liu,New sensor technology and its application [M] Beijing: Beijing University of Aeronautics and Astronautics Press, 1995.

[2] Yanbin Zhu. Latest developments and market opportunities for sensor technology [J] sensor technology, 2000: 1-3.

[3] Minsheng $\mathrm{Xu}$, Guoping Chen and other thin film physics and technology [M] Jiangsu: Southeast University Press .1987

[4] Hui Zheng.PVDF pressure sensors and other sensor technology 2003,22 (9): 5-6.

[5] Shi Ping Li .Times of the piezoelectric effect and applied basic research on the sensor actuator Heilongjiang: Heilongjiang University Press, 2014. 